\title{
HANDOVER DElay OPTIMIZATION IN PMIPV6 DOMAIN THROUGH IMPROVED UPDATE LATENCY
}

\author{
Farhana Ferdusi Liza $^{1}$ and Khan Md. Al-Farabi ${ }^{2}$ \\ ${ }^{1}$ Department of Computer Science, American International University, Dhaka, Bangladesh, \\ ${ }^{2}$ Department of Computer Science, American International University, Dhaka, Bangladesh \\ E-mail: farhana.liza@aiub.edu
}

\begin{abstract}
This paper proposes an efficient low update latency handover scheme for Proxy Mobile IPv6 (PMIPv6)-a Network-based mobility management protocol. The major aspect that introduces high latency during Mobile Node (MN) handover between Mobile Access gateways (MAGs) of the same PMIPv6 domain has been analyzed. The analysis included $M N$ profile acquirement by MAG agent and handover process between MAG agents. From this analysis, an improved solution that optimizes the handover delay has been provided. The solution has been verified by simulation in Network Simulator 2(NS2).
\end{abstract}

Keywords: PMIPv6, Handover Delay,NS2, FMIPv6, MIPv6, PMIPv6_EX

\section{Introduction}

The future is IP converged networks for multimedia applications that integrate video, voice/audio and data. The usage of wireless network has been increased considerably. The main reason behind this is, wireless technology is a truly revolutionary paradigm shift enabling multimedia communication between people and devices from any location. To support mobile operation, the backbone network has to add several new functionalities that do not exist for the wired terminal operations, because they are not usually required. These functionalities include mobility and location management, radio resource and power management and security. The very nature of mobile communication implies that the MS is constantly changing locations, warranting a need for tracking the mobile and restructuring existing connection as it moves. Mobility and location management handle the operations required for these purpose.

Handoff is the mechanism by which an ongoing connection between a MS and a correspondent terminal is transferred from one point of access of fixed network to another. Handoff management handles the massages required to make the changes in the fixed network to handle this change in the location during an ongoing communication. Location and handoff management together are commonly referred to as mobility management. Mobility management protocol can be classified as global mobility management protocol and local mobility management protocol [1].

IP, which is the most popular network layer protocol for data network, was not designed with wireless or mobile network in view. Mobile IP tries to address this issue by creating an 'anchor' for a mobile host that takes care of packet forwarding and location management. It solves the problem that IP created when the terminal is mobile. The IETF working group has proposed this Mobile IP protocol (Mobile IPv4[1-7] and Mobile IPv6[1]) as the main protocol for node mobility. Although Mobile IP technology has been standardized but is not yet implemented in the real world scenario and one of the main reason is it involves mobile node in mobility related signaling [8].

The success of WLAN switches indicates that network operators and user prefer no host stack software modification. The main reason of success in WLAN infrastructure market of WLAN switches is the capability to perform localized management without any host stack involvement. [9] WLAN switches only support WLAN networks with Ethernet backhaul and therefore are not available for advanced cellular network or pecocellular protocol or other type of wired network. So an IP-level network based localized mobility management solution is desirable. Mobile IP protocol can be used between ARs for handling local mobility, however there are 
three well known problem involved in using MIP for every movement between AR[16]. The problems are update latency, signaling overhead, location privacy.

Proxy Mobile IPv6 is a technology used to be provided for the hosts that don't need MIP stack installed to make mobility management. It is network based localized mobility protocol and the network mobility entities such as MAG and LMA are responsible for tracking the movements of the host and initiating the required mobility signaling on its behalf. The main goal of PMIP protocol is to provide Mobility Management without host involvement and give batter performance in handoff process to make multimedia communication efficient. In this paper the PMIPv6 protocol has been investigated according to the IETF specification [6]. A performance enhancement method has been proposed as a scope of improvement has been recognized in handover process which will make multimedia communication in all IP network evenhanded. The performance has been measured for multimedia communication and compared against PMIP.

This paper is structured in the following manner: section II will describe the related work and the following section will detail the PMIPv6 protocol in general, section IV will highlight the handover process between MAG agents in standard PMIP domain, and the proposed extension has been illustrated in section V. The implementation and simulation technique to verify the proposed protocol extension has been shown in section VI and VII. Network Simulator 2 (NS2) was used to do the simulation. Profiling and experimentation with the proposed design shows that it is lightweight and performs smoothly without causing extensive overhead.

\section{Related Work}

In IP networks the main problem with node mobility is that the IP address functions both as the identifier as well as the locator of a MN in the routing hierarchy. In its role as identifier, the IP address has to be fixed in order to allow the MN to be identified by Correspondent Nodes (CNs) that want to initiate communication with it, and also because it is used by MNs to identify their ongoing Transmission Control Protocol (TCP) connections. In its role as locator, the IP address of a MN has to change when it moves to a different sub-network, in order not to disrupt the route aggregation in the Internet. IP-level mobility solutions address this problem by a new Protocol called Mobile IP .[1]

IETF proposed Mobile IP protocol enables mobile computers to roam seamlessly in different administrative domains. However, the protocol has its disadvantages when mobile hosts rate of handoff increases within the foreign administrative domain and from one foreign domain to another and uses $\mathrm{MN}$ to perform mobility management. A number of approaches have been taken in order to reduce the handoff latency.

One approach taken was the introduction of micro mobility protocols which were broadly aimed at improving the transparent roaming of mobile hosts at the subnet level of a network domain. A number of these solutions have been proposed since the introduction of Mobile IP. Campbell [7] has written a survey of micro-mobility protocols.

Further to this, an IETF working group, called Seamoby, was formed to resolve the complex interaction of parameters and protocols needed for seamless handoffs. The two main issues being dealt by this working group are the dormant mode host alerting problem (i.e. paging) [8], and context transfers between nodes in an IP access network (i.e. handoff) [9]. The work proposed in this paper can complement micro-mobility protocols and Seamoby efforts, introducing minimal additions to the mobile host for less signaling overheads.

The IETF MobileIP working group has an Internet Draft proposing a protocol for supporting fast handoffs: FMIPv6[10]. The protocol aims to reduce the handoff latency caused by the movement detection and the Mobile IP registration process. When a handoff is imminent, the later problem is solved by keeping the mobile host ongoing traffic alive with the current access router while the Mobile IP registration process is carried out with the new access router. Like the MIPv6 draft, FMIPv6 also broadly suggests a trigger for the "Handoff Initiation" which may derive from specific link layer (L2) events or policy rules.

A number of simple methods have been proposed, some modifying network entities, which directly tackle the handoff latency in Mobile IP. These are based on hierarchical or 
multicast handoff mechanisms. C`aceres[11] obtained experimental results for the performance of a minimal hierarchical handoff scheme. This had the advantages of not having the complexity of extending routes or anticipating handoffs to improve the handoff latency. A simple multicast technique proposed by Helmy [12] suggested minor modifications to Mobile IP, and validated the method by simulation.

All of the technique described in this section requires the MN's participation in Signaling to deal with the mobility. There are certain problems that are associated with that issue have been described in previous section. Furthermore, In MIPv6 [13] binding updates are sent from the $\mathrm{MN}$ to the $\mathrm{CN}$ with every move. Although this alleviates the triangle routing problem in MIP, the communication overhead is still high during handoff rendering MIP unsuitable for micro mobility and causing it to be inadequate for multimedia communication.

The IETF NETLMM working group has proposed Proxy mobile IPv6 which is able to manage localized micro mobility without the necessity of $\mathrm{MN}$ in signaling process. Although PMIPv6 solves the deployment problem associated with MN signaling participation in mobility management, there are some problems has been addressed in recent research work. The challenge in NGWN mobility management is to reduce handover delay. There are some effort that has been made to solve these problem particularly to reduce handover latency in[14]. Although the handover latency was improved but this process has used the MN in the management procedure and which is not actually what we are looking for. In [15] a low latency handover scheme was proposed which utilize Media Independent Handover (MIH). By using MIH services a solution is provided that minimizes overall scanning delay at Layer 2, the information service provided in MIH can provide context transfers, and efficient triggering events can speed up handover procedure. However, if only L2 handover delay is optimized, it will not give very efficient performance until L3 handover delay is also optimized as the overall handover delay (L2 + L3) is insignificant for demanding and delaysensitive applications.
In this paper, an approach has been considered to reduce the handover delay using a novel method which utilizes multicast massage in PMIP domain. This development extends the original PMIPv6 protocol. This process also includes a buffer storage which significantly reduces the packet loss.

\section{Proxy Mobile IPv6 Protocol}

The PMIPv6 architecture consist of three entity: Local Mobility Anchor (LMA), Mobile Access Gateway (MAG) and Mobile node(MN). The Local Mobility Anchor (LMA) is equivalent to MIPv6 Home Agent (HA) [1] with additional capability, which maintains the address bindings for mobile nodes (MN) in the PMIPv6 domain. The Mobile Access Gateway (MAG) is a new and additional entity (the proxy agent for MN), which actually do the mobility related signaling on behalf of the mobile node which previously used to do by the MN itself in MIPv6. Mobile nodes themselves are not required to provide any additional function compared to basic IPv6 specifications [3, 4].

According to PMIPv6 specification if network determines that the mobile node is authorized for network based mobility service, the network will ensure that the mobile node will be able to obtain the address configuration on the connected interface. Through the address configuration MN actually get home network prefix (es), the default router address on the link and other address configuration parameter. The MAG emulate the foreign link with the MN's home network, such that the MN always sees its home prefix wherever it connect in any link in PMIPv6 domain and always configures the same address . By this mechanism, mobility can successfully be concealed from the terminal, therefore PMIPv6 solved one of the important deployment problem for localized mobility management.

In PMIPv6 domain, although the multiple mobile nodes can share same address prefix, stateless address autoconfiguration put constraint on in this case so per-MN-prefix is considered in this paper to define the architecture. Whenever an MN try to enter the operator's network, it performs a layer 2 network entry procedure, at same stage it is authenticated by a AAA server (policy profile). If the $\mathrm{MN}$ is authorized to receive proxy mobility services, the MAG retrieves 
the MN's profile from the policy profile, which contains the MN's home network prefix and permitted address configuration modes. After reception of this profile, the MAG sends a Proxy Binding Update to the LMA, containing the MN identifier and its home network prefix. The MAG's IP address is set as the proxy Care-Of-Address. The LMA sets up a binding for the MN's home network prefix to the MAG's IP address with a lifetime period. To emulate the MN's home network, the MAG starts sending Router Advertisements (RA) advertising the MN's home network prefix. If the MN is IPv6 capable it might have solicited an RA by sending a Router Solicitation message. After reception of the Proxy Binding Acknowledgment, the MAG completes the bidirectional tunnel establishment and configures its routing table accordingly. All traffic from and to the $\mathrm{MN}$ can now be forwarded through the tunnel between MAG and LMA.

\section{Handover Process between MAG Agents of a PMIPv6 Domain}

PMIPv6 local handoff occurs when a MN moves beyond the radio range of one MAG of the PMIP domain and enters into another MAG range. In this paper we will only consider the intra-domain handoff situation.

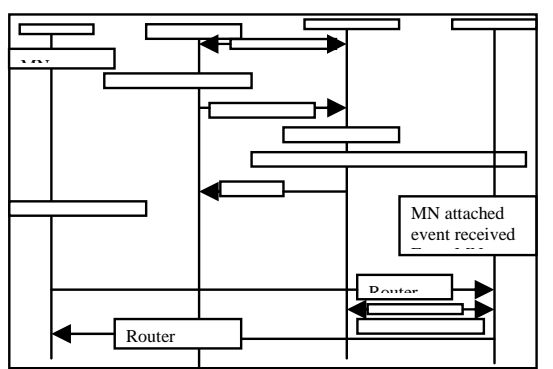

Fig. 1: Signaling call flow of MN handoff

Fig. 1 shows the signaling call flow for the mobile node's handoff from the previously attached mobile access gateway (p-MAG) to the newly attached mobile access gateway (nMAG).

According to PMIPv6 specification [6] the present handover process between MAGs of the same domain can be summarized as follows: after obtaining the initial address configuration in the Proxy Mobile IPv6 domain, if the mobile node changes its point of attachment, the mobile access gateway on the previous link will detect the mobile node's detachment from the link. It will signal the local mobility anchor and will remove the binding and routing state for that mobile node. The local mobility anchor, upon receiving this request, will identify the corresponding mobility session for which the request was received, and accepts the request after which it waits for a certain amount of time to allow the mobile access gateway on the new link to update the binding. However, if it does not receive any Proxy Binding Update message within the given amount of time, it will delete the binding cache entry.

The mobile access gateway on the new access link, upon detecting the mobile node on its access link, will signal the local mobility anchor to update the binding state. After completion of the signaling, the serving mobile access gateway will send the Router Advertisements containing the mobile node's home network prefix(es), and this will ensure the mobile node will not detect any change with respect to the layer-3 attachment of its interface.

\section{Proposed Extension to PMIPv6 protocol}

The current process of handover described above is better in performance compared to MIPv6 and this has been shown in [4]. However, the PMIPv6 handover delay is still higher than the standard of IETF [6].Although there will be always a handover delay, but the optimization of the process will make the real time application feasible in mobile environment.

From the discussion of the previous section we have seen that the LMA has to wait for a certain amount of time to allow the MAG on the new link to update the binding. During this time, if the MN were in the middle of active connection with a $\mathrm{CN}$ node than there will be an interruption in data reception which will be a cause of packet loss and performance digression.

To minimize the interruption when a MAG in a PMIPv6 domain recognized a MN (when the MN enter into the domain for the first time), the MAG will do the signaling according to the PMIPv6 specifications [6]. In the rest of this paper we will cal this MAG as First MAG of the domain. This will also ensure that whether $\mathrm{MN}$ is authorized for that PMIPv6 domain or not. After that the MN will configure its address in the access link. 
The MAG will then send a Multicast massage (containing MN_Identifier ) to all other MAG of that domain, as a result corresponding MAGs will be able to acquire MN profile of that $\mathrm{MN}$ even though the $\mathrm{MN}$ is not yet connected on their access link. The concept behind this is if $\mathrm{MN}$ is authorized for the particular domain, each MAG of the domain will get the same authentication result. Therefore the time to authenticate a MN again and again on each movement of MN from one MAG to other for a domain and the time to acquire the profile of a $\mathrm{MN}$ afterward will be saved. How each MAG will recognize other MAGs of the domain as a multicast group will be depend on particular implementation.

However general implementation technique can be described with the following example, if the particular PMIP domain contains five MAG agents and if these agents

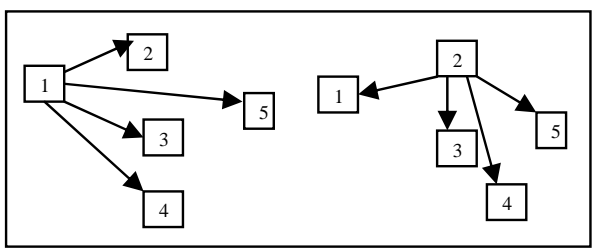

Fig. 2: .Multicast group assignment

were numbered numerically as in fig. 2 then MAG agent 1 will see MAG agent $\{2,3,4,5\}$ as a multicast group, in the same manner MAG agent 2 will see MAG agent $\{1,3,4,5\}$ as a multicast group and so on. Now if the perspective MAG which has already acquired profile on response of the multicast massage, receive Router Solicitation massage from the MN or detect that particular MN is being attached to its access link, it can send PBU to LMA straight way as first two steps have already been executed before the $\mathrm{MN}$ has actually physically get attached to the link.

In this way the waiting period of LMA from receiving the deregistration request on recognizing the detachment of $\mathrm{MN}$ from previous link to reception of PBU from new MAG will be minimized. Furthermore as the waiting period has been minimized by the proposed scheme, LMA can keep a buffer to temporarily store data from $\mathrm{CN}$ when $\mathrm{MN}$ is not attached to any MAG. The concept behind keeping a buffer is as it is expected that the waiting period is very low there is very little chance that the data received will run out the buffer and LMA will discard the packet and make an issue of packet loss.

When a LMA receives a deregistration PBU from a MAG; and if the MN was in an active connection with a $\mathrm{CN}$ at that time, the LMA will store these data in a buffer for a certain amount of time which will be determined by the 'MinDelayBeforeBCEDelete' parameter of PMIPv6 protocol. If the LMA receives any PBU from MAG agent then LMA will forward those data from buffer and this way the packet loss can be minimized.

This approach has given a better handover performance and low packet loss which has been examined by using NS2 simulation.

\section{Implementation}

The implementation architecture of PMIPv6 [4] was used to implement the proposed handover process with following extension. In [4], there is one kind of MAG agent, however in our proposed scheme the MAG agents are two types based on their operation. The First MAG which is a typical PMIPv6 MAG agent and rest of the MAG agents which have little modification according to our proposed scheme.

For MAG agents of a domain the signalling call flow between MN, LMA and MAG is as follows:

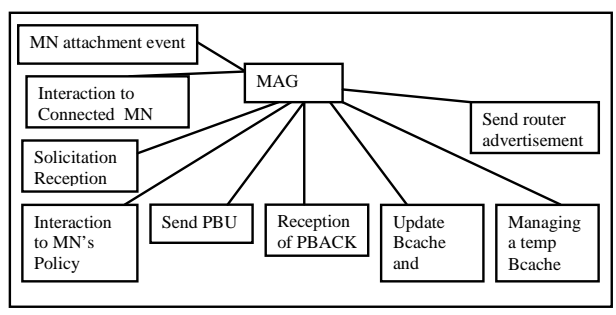

Fig. 3: First MAG's signalling call flow

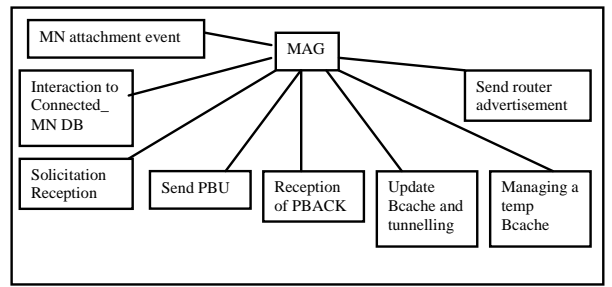

Fig. 4: MAG agents' signaling call flow except the first MAG

From the above figure it is apparent that for the MAG agents except the first one who will do signalling according to PMIPv6 specification don't need the "Interaction to MN's Policy” step.

The data structure of connected_MN DB:

The Connected_MN database can be implemented as a structure array.

Struct Connected_MN

\{ 
int MN_ID,

struct MN_Profile *next

\}con_mn[NO_OF_MN];

The interface relation among the MAG objects are shown in following figure

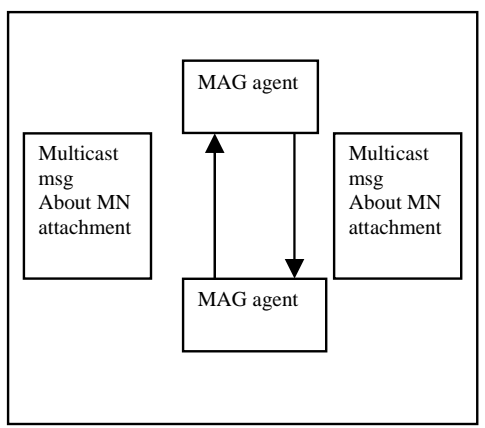

Fig. 5: Interface relationship (MAG to MAG)

The interface relation among the MAG and other objects are shown in following figure

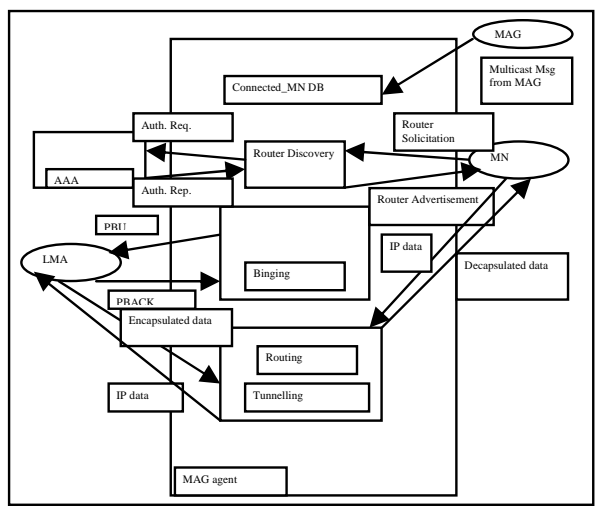

Fig. 6: Interface relationship (MAG to other agent)

The proposed handover process requires a new type of mobility packet extension whose format is as follows

\begin{tabular}{|l|c|}
\cline { 2 - 3 } \multicolumn{1}{|l|}{ Typ } & $\begin{array}{c}\text { Len } \\
\text { M }\end{array}$ \\
\hline \multicolumn{2}{|l|}{ Multicast Set ID } \\
\hline
\end{tabular}

Type $=8$ Bits

Length $=8$ bits

Multicast Set ID=16 Bits

Fig. 7: Multicast Packet Header Extension

\section{Simulation}

Using the Network Simulator 2 (NS-2) simulation software, we simulated a mobile node moving into a PMIPv6 domain and thus observed the initial attachment and then mobile node was moving from one MAG coverage to another MAG coverage of the same PMIPv6 domain. Fig. 8 shows the simulation scenario that we have used. In the figure, Mobile Node started its movement and entered into PMIPv6 domain and first it registered with MAG1, which is connected to a wired node LMA. The Mobile Node will see this access link as a home link as the MAG will do router advertisement with MN's Home Network prefix.

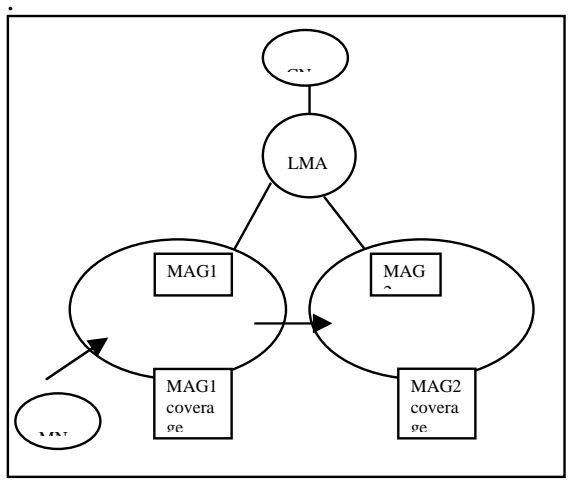

Fig. 8: Simulation scenario

The $\mathrm{CN}$ is sending jitter to $\mathrm{MN}$. During 20 second simulation, MN moved into MAG1 coverage area at time $=2 \mathrm{~s}$, to MAG2 at time $=12 \mathrm{~s}$ and moving out of the MAG2 coverage area at time $=18 \mathrm{~s}$.

Two different simulations were carried out with the same scenario. In the first simulation, MAG agents did not use the Profile sharing scheme. In the second simulation, the MAG agents did use the profile sharing scheme which has been named as PMIP_EX. Handover delay in both case has been shown in following figure.

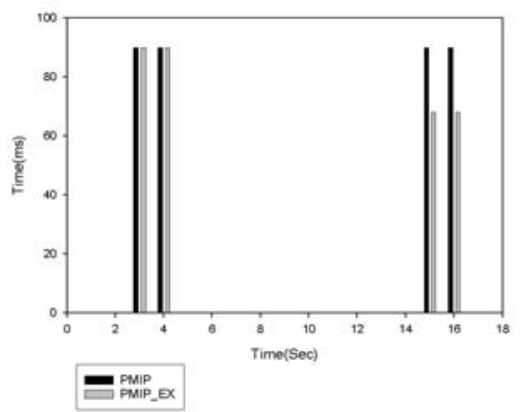

Fig. 9: Hanover delay comparison between PMIP and PMIP_EX

From the above figure it has been shown that the profile sharing scheme gave better performance than the conventional PMIPv6 protocol. For the first attachment of the $\mathrm{MN}$ 
to the PMIPv6 domain gave same handover delay in PMIPv6 extension as PMIPv6, however when MN moved to another MAG of the same domain, that time handover delay was almost half of the conventional mechanism.

Although the proposed scheme gave batter performance in intra-domain handover, the additional signaling introduced specially for the multicast signaling, made a issue of bandwidth waste which has been shown in the following figure for the previous simulation scenario. From the figure it is obvious that signaling overhead has been increased from 11100 (aprx.) to 12050 (aprx.) which is $0.095 \%$ increment of overhead with reference to the original data. Moreover this over head will only effect the local domain not the whole infrastructure network.

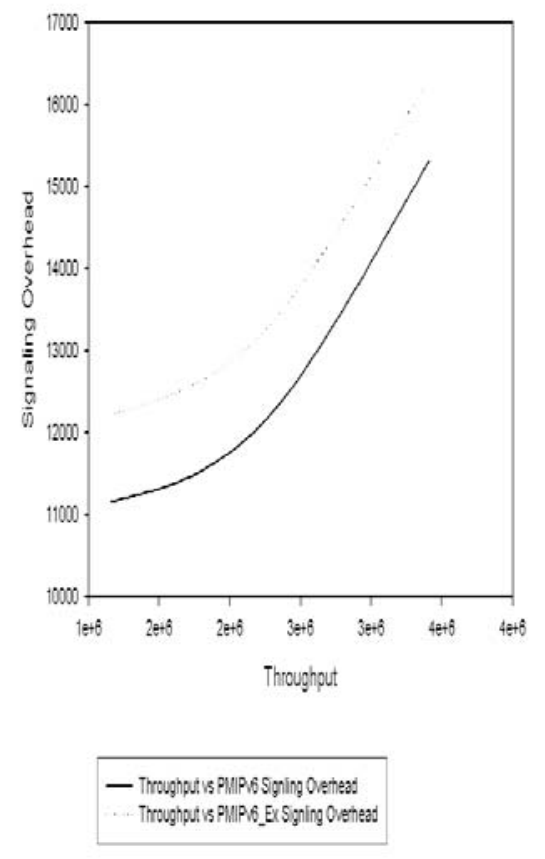

Fig.10: Comparison of signaling overhead between PMIP and PMIP_EX

Table 1: Comparison of signaling overhead between PMIP and PMIP_EX

\begin{tabular}{|l|l|l|l|}
\hline $\begin{array}{l}\text { Packet } \\
\text { Size }\end{array}$ & Throughput & $\begin{array}{l}\text { PMIPv6 } \\
\text { Signling } \\
\text { Overhead }\end{array}$ & $\begin{array}{l}\text { PMIPv6_E } \\
\text { x Signling } \\
\text { Overhead }\end{array}$ \\
\hline 1500 & 1154736 & 11157 & 12223 \\
\hline 1000 & 2254736 & 12157 & 13259 \\
\hline 500 & 3409472 & 15321 & 16325 \\
\hline
\end{tabular}

Although the proposed scheme introduce a slight increase of signaling overhead, as in the proposed scheme a buffer was kept by the LMA, the packet loss was reduced significantly which has been shown in the following figure.

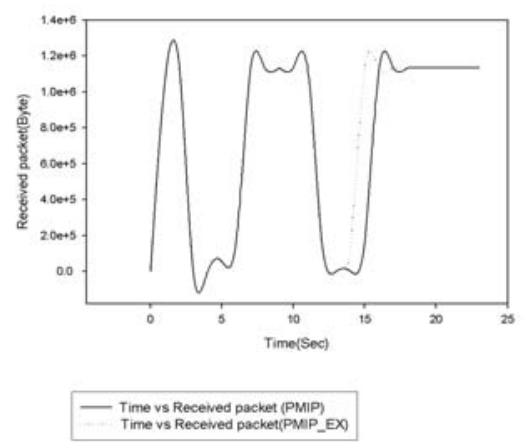

Fig. 11: Comparison of packet loss between PMIP and PMIP_EX

Based on the above discussion it is apparent that the default registration process where the MAG sends a authentication massage to AAA server on recognizing a new MN has attached to its network or after received the solicitation massage from MN is inefficient or suboptimal as the MAG has to check whether the MN is authorized or not on each attachment of the MN to different links in the same domain. These authentication massage delay the process of handover and make an issue of packet loss which eventually make multimedia communication infeasible in ALL-IP mobile environment. The proposed scheme has improved the process by using a novel registration process which reduces the update latency in the case of intra-domain handover between MAG agents.

\section{Conclusion}

In this paper we proposed a novel low update latency handover process where time optimization is done in authentication and profile acquirement phase. Our proposed approached has been demonstrated using NS2 and it gave better performance in handover process than normal PMIPv6 protocol.

\section{References}

[1] D. Johnson, C. Perkins, and J. Arkko, "Mobility Support in IPv6,”RFC 3775, June 2004.

[2] Kempf, J., "Problem Statement for NetworkBased Localized Mobility Management (NETLMM)", RFC 4830, April 2007. 
[3] Official website for Network-based Localized Mobility Management (netlmm) http://www.ietf.org/html.charters/netlmmcharter.html

[4] Liza, Farhana F.; Yao, Wenbing.; " Architecture of Proxy Mobile IPv6 Protocol and its implementation for NS2 Simulator software" IEEE International Conference on communication software and networks (ICCSN 2009).

[5] Official website for Network Simulator 2.http://www.isi.edu/nsnam/ns/

[6] S. Gundavelli, Ed., K. Leung, V. Devarapalli, K. Chowdhury, B. Patil," Proxy Mobile IPv6”, RFC 5213,August 2008.

[7] A. Campbell and J. Gomez-Castellanos. IP Micro-Mobility Protocols. ACM SIGMOBILE Mobile Computing and Communications Review, 4(4):45-53, October 2000.

[8] J. Kempf. Dormant Mode Host Alerting (IP Paging) Problem Statement. IETF Request for comments, RFC 3132,June 2001.

[9] J. Kempf. Problem Description: Reasons For Doing Context Transfers Between Nodes in an IP Access Network. IETF Request for comments, RFC 3374, September 2002.

[10] R. Koodli. Fast Handovers for Mobile IPv6. IETF Internet- Draft, September 2002.
[11] R. C'aceres and V. Padmanabhan. Fast and scalable wireless handoffs in supports of mobile Internet audio. Mobile Networks and Applications, 3(4):351-363, 1999.

[12] A. Helmy. A multicast-based protocol for ip mobility support. In Proceedings of NGC 2000 on Networked Group Communication, pages 49-58. ACM Press, 2000.

[13] C. Perkins and D. Johnson, "Mobility Support in IPv6", Proceedings of MobiCom'96, November 1996.

[14] Geert Heijenk, Mortaza S. Bargh, Julien Laganier, and Anand R. Prasad,"Reducing Handover Latencyin Future IP-based Wireless Networks:Fast Proxy Mobile IPv6”.

[15] Kim I.,Chul Jung Y., Kim Y."Low Latency Proactive Handover Scheme for Proxy MIPv6 with MIH”, Proceedings of the 11th AsiaPacific Symposium on Network Operations and Management: Challenges for Next Generation Network Operations and Service Management .

[16] J. Kempf, Ed.," Problem Statement for Network-Based Localized Mobility Management (NETLMM)”,RFC 4830, April 2007.

[17] C. Perkins, “IP Mobility Support for IPv4,"RFC 3344, August 2002. 